\title{
Recovery of directed graphs from the matrix of peaks neighborhood
}

\author{
Kotenko A.P., \\ Samara State Aerospace University \\ Dokuchaev A.V. \\ Samara State Technical University
}

\begin{abstract}
The properties of the graph problem of optimal investment of additional resources available to reduce the critical path network project are considered. It was proposed the algorithm in graph structure of the project on a given matrix of precedence works. The specified method of minimizing the required number of fictitious works to simplify the graph of the project is indicated.
\end{abstract}

Keywords: problem of network planning and management, lists of predecessors, graph of the project, minimizing of the required number of fictitious works

Citation: Kotenko A.P., Dokuchaev A.V. Recovery of directed graphs from the matrix of peaks neighborhood. Proceedings of Information Technology and Nanotechnology (ITNT-2015), CEUR Workshop Proceedings, 2015; 1490: 406-413. DOI: 10.18287/1613-0073-2015-1490-406-413

\section{Statement of the problem}

Continued [1-4] to study the problem of network planning and management for the connected project $P=\{a(i)\}_{i=1}^{k \geq 1}$ with the jobs

$a(i): i \neq j \Leftrightarrow a(i) \neq a(j)$

which has the sets of predecessors $s(a(i)) \subset P$ and execution time

$t(a(i), x(i)): P \times U(X) \rightarrow \mathfrak{R}^{+}$

by additional resources $x(i) \in U(X)$ of the partition

$\left.U(X) \stackrel{\text { def }}{=}\{x(i)\}_{i=1}^{k}: X=\bigcup_{i=1}^{k} x(i), x(i) \bigcap_{i \neq j} x(j)=\varnothing\right\}$.

Here $s(a(i))$ is own (may be empty) subset of project jobs $P$, which must be completed before starting job $a(i)$. 
Resource $X$ can be a real number with a non-negative partitioning $0 \leq \sum_{i=1}^{k} x(i) \leq X \quad\left(\right.$ or $\left.1<\prod_{i=1}^{k} x(i) \leq X\right)$, or the set of discrete elements $X=\left\{y_{t}\right\}_{t=1}^{L \geq 1}$ with arbitrary partition into subsets $U(X) \subseteq 2^{x}$.

Let optimize the resource allocation $\{x(i)\}_{i=1}^{k}$ for minimizing the total time of the project $P: \sum_{i=1}^{k} t(a(i), x(i)) \rightarrow \min$

\section{Reduce the list of predecessors}

We describe the relation $S$ of precedence $a_{i} S a_{j}$ of the project jobs $a_{i}, a_{j} \in P$ by the matrix of precedence $S=\|s(i, j)\|_{i, j=1}^{k}$ :

$s(i, j) \stackrel{\text { def }}{=} \chi(a(i), s(a(j)))$

with the characteristic function $\chi(a(i), s(a(j))) \stackrel{\text { def }}{=}\left\{\begin{array}{l}1 \leftarrow a(i) \in s(a(j)), \\ 0 \leftarrow a(i) \notin s(a(j)) .\end{array}\right.$

Thus, the unit $l$ in the $i$-th row of the matrix $S$ indicates preceding the job $a(i)$ of the respective columns. Then the transposed matrix $S^{T}$ describes the inverse relation $S^{*-1}$ following $a_{j} S-1 a_{i}$ project job: unity $l$ in the $i$-th row of the matrix $S^{T}$ indicates preceding job $a(i)$ by the job of relevant columns. Obviously, the relations $\mathrm{S}$ and $S^{T}$ are transitive ( $S^{2} \subseteq S,\left(S^{-1}\right)^{2} \subseteq S^{-1}$ ) through the finiteness of the set of project jobs and has the inclusions

$$
\begin{aligned}
& \varnothing=S^{k-1} \subseteq S^{k-2} \subseteq \ldots \subseteq S^{3} \subseteq S^{2} \subseteq S \\
& \varnothing=\left(S^{-1}\right)^{k-1} \subseteq\left(S^{-1}\right)^{k-2} \subseteq \ldots \subseteq\left(S^{-1}\right)^{3} \subseteq\left(S^{-1}\right)^{2} \subseteq S^{-1} .
\end{aligned}
$$

We call the job $a(i) \in P$ immediate predecessor of the job $a(j) \in P$, if $\chi(a(i), s(a(j)))=1$ and no other job such as $a(l) \in P: \chi(a(i), s(a(l)))=\chi(a(l), s(a(j)))=1$.

Otherwise, the preceding (following) will be called indirect.

With the immediate predecessors lists $\left\{s^{*}(a(i))\right\}_{i=1}^{k}$ we define the relation $S^{*}$ of immediate precedence by $k \times k$-matrix of direct precedence $S^{*}=\left\|s^{*}(i, j)\right\|_{i, j=1}^{k}$ :

$s^{*}(i, j) \stackrel{\operatorname{def}}{=} \chi\left(a(i), s^{*}(a(j))\right)$.

Let the transposed matrix $S *^{T}$ defines the inverse relation $S *^{-1}$ of immediately following project jobs. Wherein the relations $S^{*}$ and $S^{*^{-1}}$ are generally transitive as

$$
S^{*}=S-\bigcup_{t=2}^{k-1} S^{t} \text { and } S^{*-1}=S^{-1}-\bigcup_{t=2}^{k-1}\left(S^{-1}\right)^{t} \text {. }
$$


We shorten the list of precedence $\{s(a(i))\}_{i=1}^{k}$ to the list of direct precedence $\{s *(a(i))\}_{i=1}^{k}$ and by permutation of rows and columns of the matrix $S$ for correct precedence order of the project jobs:

$\chi(a(j), s *(a(i))) \underset{i<j}{=} 0$.

In the future, we consider the original numbering of project jobs $\{a(i)\}_{i=1}^{k}$ is correct, and the original list of predecessors $\{s(a(i))\}_{i=1}^{k}$ is shorthand. Let matrix $A$ of direct precedence of the properly ordered jobs

$S=\left(A\left[i_{1} \times j_{1}\right], A\left[i_{2} \times j_{2}\right], \ldots, A\left[i_{r} \times j_{r}\right]\right), \quad 2 \leq r \leq k-1, \quad i_{1}=j 1=i 2, \quad j 2=i 3, \quad \ldots, \quad j_{r}-1=i r=j r$, $\sum_{t=2}^{r} i_{t}=\sum_{t=1}^{r-1} j_{t}=k$

be upper triangular and block-chained with zeros on the main diagonal and has continuous chain $i_{t} \times j_{t}$-blocks $A\left[i_{t} \times j t\right], 1 \leq t \leq r$, of which the first block and the last block are zero and square

$A\left[i 1 \times j_{1}\right]=0, A\left[i_{r} \times j_{r}\right]=0$.

Let the remaining blocks are non-zero: $A\left[i_{t} \times j t\right] \neq 0,2 \leq t \leq r-1$.

\section{Example 1.}

The matrix of direct precedence of properly ordered 9 project jobs $\{a(i)\}_{i=1}^{9}=P$ may take the form

\begin{tabular}{|c|c|c|c|c|c|}
\hline & $a(1) \vdots a(2)$ & $a(3) \vdots a(4) \vdots a(5)$ & $a(6) \vdots a(7)$ & $a(8) \vdots a(9)$ & \\
\hline $\begin{array}{l}a(1) \\
a(2)\end{array}$ & $A\left[i_{1} \times j_{1}\right]$ & $A\left[i_{2} \times j_{2}\right]$ & 0 & 0 & $i_{2}$ \\
\hline $\begin{array}{l}a(3) \\
a(4) \\
a(5)\end{array}$ & 0 & 0 & $A\left[i_{3} \times j_{3}\right]$ & 0 & $i_{3}$ \\
\hline $\begin{array}{l}a(6) \\
a(7)\end{array}$ & 0 & 0 & 0 & $A\left[i_{4} \times j_{4}\right]$ & $i_{4}$ \\
\hline $\begin{array}{l}a(8) \\
a(9)\end{array}$ & 0 & 0 & 0 & $A\left[i_{5} \times j_{5}\right]$ & $i_{5}$ \\
\hline & $\underbrace{-}_{j_{1}}$ & $\underbrace{}_{j_{2}}$ & $\underbrace{-}_{j_{3}}$ & $\underbrace{}_{j_{4}}$ & \\
\hline
\end{tabular}

\section{Project's graph}

We construct a directed graph $G(V, R)$ of the project $P$. At first we define the set of vertices $V$ as follows.

Case 1. If block $A[i t \times j t], 2 \leq t \leq r-1$, consists only of units, then exists a general conclusion

$v_{\kappa}(a(\alpha(1)))=v_{\kappa}(a(\alpha(2)))=\ldots=v_{\kappa}(a(\alpha(i t)))$

and common origins

$v_{H}(a(\beta(1)))=v_{H}(a(\beta(2)))=\ldots=v_{H}\left(a\left(\beta\left(j_{t}\right)\right)\right)$ 
of the project jobs $a(\alpha(1)), a(\alpha(2)), \ldots, a(\alpha(i t)), a(\beta(1)), a(\beta(2)), \ldots, a(\beta(j t)) \in P$ with numbers

$1 \leq \sum_{s=1}^{t-1} i_{s}=\alpha(1)<\alpha(2)<\ldots<\alpha\left(i_{t}\right)<\beta(1)<\beta(2)<\ldots<\beta\left(j_{t}\right)=\sum_{s=t+1}^{r} j_{s}$.

Wherein

$\alpha(1)=\sum_{s=1}^{t-1} i_{s}, \quad \alpha(s+1)=\alpha(s)+1, \quad 1 \leq s \leq i_{t}-1 ; \quad \beta(1)=\alpha\left(i_{t}\right)+1, \quad \beta(s+1)=\beta(s)+1, \quad 1 \leq s \leq j t-1$,

$\beta\left(j_{t}\right)=\sum_{s=1}^{t} i_{s}+j_{t}$

We may add vertex $v(\alpha(1), \alpha(2), \ldots, \alpha(i t) ; \beta(1), \beta(2), \ldots, \beta(j t)) \in V$ to connect said project jobs (Fig. 1). In this way,

$v(\alpha(1), \alpha(2), \ldots, \alpha(i t) ; \beta(1), \beta(2), \ldots, \beta(j t))=$

$=v_{\kappa}(a(\alpha(1)))=v_{\kappa}(a(\alpha(2)))=\ldots=v_{\kappa}(a(\alpha(i t)))=v_{H}(a(\beta(1)))=v_{H}(a(\beta(2)))=\ldots=v_{H}\left(a\left(\beta\left(j_{t}\right)\right)\right)$.

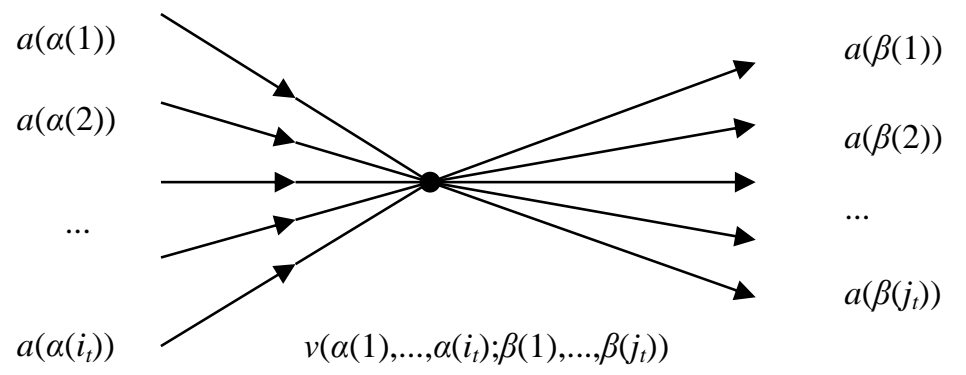

Fig. 1. - Top, obtained by combining inbound and outbound directed arcs

One can consider each added vertex $v(\alpha(1), \ldots, \alpha(i t) ; \beta(1), \ldots, \beta(j t))$ as notation of the complete bipartite graph $K(i t, j t), 1 \leq i_{t} \leq k-1,1 \leq j_{t} \leq k-1$, with the part $K\left(1, i_{t}\right)$ powered of $i t$ and the part $K(j t, 1)$ powered of $j t$, having single $i_{t} \times j_{t}$-, $l \times i_{t}$ - and $j_{t} \times 1$-neighborhood matrix peaks co-responsible. Shares of $K(1, i t)$ and $K(j t, 1)$ describe the overall time of completion

$K\left(1, i_{t}\right)=\left\{v_{\mathrm{K}}(a(\alpha(s)))\right\}_{s=1}^{i_{t}}$

and common moments of the beginning

$K\left(j_{t}, 1\right)=\left\{v_{\mathrm{H}}(a(\beta(s)))\right\}_{s=1}^{j_{t}}$

of the relevant design jobs. See. Fig. 2.

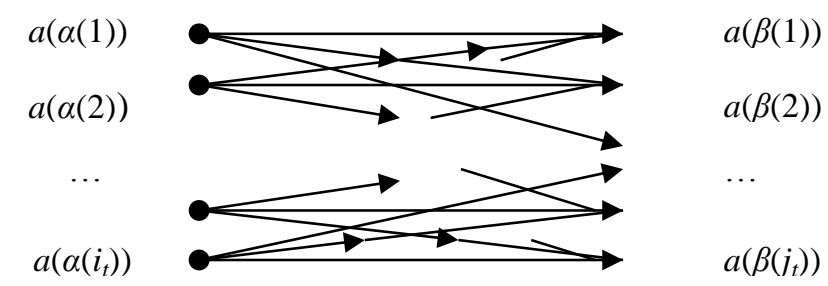

Fig. 2. - Key added in the form of the vertices bipartite graph 
Obviously that the resulting subgraph $G\left(A\left[i_{t} \times j_{t}\right]\right)$, which connects the project jobs $\{a(\alpha(u))\}_{u=1}^{i_{t}} \cup\{a(\beta(u))\}_{u=1}^{j_{t}}$,

is planar, and the number of options for passing full paths through project $P$ unit block $A\left[i_{t} \times j_{t}\right]$ is equal to $i_{t} \times j_{t}$.

Case 2. If among the elements of the block $A\left[i_{t} \times j_{t}\right], 2 \leq t \leq r-1$, there are zeros, then relevant bipartite graph $K(i t, j t)$ is not complete and must be added by the fictional jobs, unlike the embodiment of Fig. 1 it can not to connect a it incoming and jt outgoing directed arcs by one vertex.

\section{Example 2.}

Consider the $2 \times 2$-blocked $5 \times 9$-matrix of the immediate preceding project jobs $\{a(i)\}_{i=6}^{14}$ by the jobs $\{a(i)\}_{i=1}^{5}$ :

$A[5 \times 9]=\left(\begin{array}{cccc:ccccc}1 & 1 & 1 & 1 & 1 & 1 & 1 & 1 & 1 \\ 1 & 1 & 1 & 1 & 1 & 1 & 1 & 1 & 1 \\ 1 & 1 & 1 & 1 & 1 & 1 & 1 & 1 & 1 \\ \hdashline 1 & 1 & 1 & 1 & 0 & 0 & 0 & 0 & 0 \\ 1 & 1 & 1 & 1 & 0 & 0 & 0 & 0 & 0\end{array}\right)$.

Combining events (Fig. 3)

$v_{\kappa}(a(1))=v_{\kappa}(a(2))=v_{\kappa}(a(3))=v_{H}(a(10))=v_{H}(a(11))=v_{H}(a(12))=v_{H}(a(13))=v_{H}(a(14))$,

$v_{\kappa}(a(4))=v_{\kappa}(a(5))=v_{H}(a(6))=v_{H}(a(7))=v_{H}(a(8))=v_{H}(a(9))$,

introduce the additional (fictional) job $b(1)$ to save the immediate preceding of the jobs $\{a(i)\}_{i=6}^{9}$ by the jobs $\{a(i)\}_{i=4}^{5}$, and the preceding of the jobs $\{a(i)\}_{i=10}^{14}$ by the jobs $\{a(i)\}_{i=1}^{3}$, and non-immediate preceding of the jobs $\{a(i)\}_{i=6}^{9}$ by the jobs $\{a(i)\}_{i=1}^{3}$.

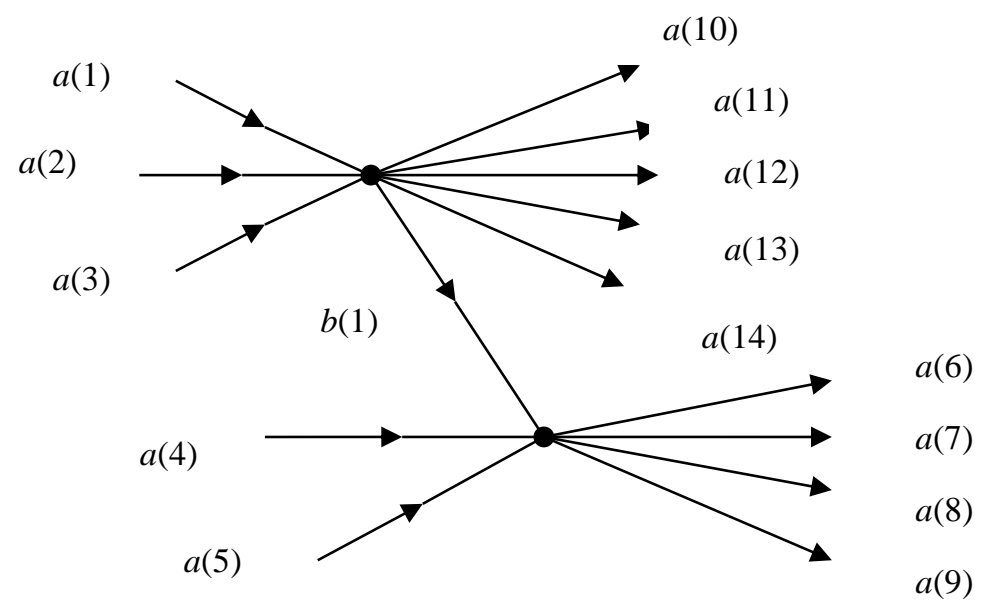

Fig. 3. - A graph constructed according to Example 2 
The permutation of the project jobs $\{a(i)\}_{i=6}^{9}$ and $\{a(i)\}_{i=10}^{14}$ and the addition of the fictional job $b(1)$ between $a(5)$ and $a(10)$ does not change the other blocks of the matrix of directly precedence $S$, setting the proper ordering of a united set of jobs $b(1) \cup\{a(i)\}_{i=1}^{14}$ and turning the block $A[5 \times 9]$ to the $2 \times 2$-blocked $6 \times 10$-submatrix

\begin{tabular}{|c|cccccc:cccc|}
\hline & $b(1)$ & $a(10)$ & $a(11)$ & $a(12)$ & $a(13)$ & $a(14)$ & $a(6)$ & $a(7)$ & $a(8)$ & $a(9)$ \\
\hline$a(1)$ & 1 & 1 & 1 & 1 & 1 & 1 & 0 & 0 & 0 & 0 \\
$a(2)$ & 1 & 1 & 1 & 1 & 1 & 1 & 0 & 0 & 0 & 0 \\
$a(3)$ & 1 & 1 & 1 & 1 & 1 & 1 & 0 & 0 & 0 & 0 \\
\hdashline$a(4)$ & 0 & 0 & 0 & 0 & 0 & 0 & 1 & 1 & 1 & 1 \\
$a(5)$ & 0 & 0 & 0 & 0 & 0 & 0 & 1 & 1 & 1 & 1 \\
$b(1)$ & 0 & 0 & 0 & 0 & 0 & 0 & 1 & 1 & 1 & 1 \\
\hline
\end{tabular}

with two $3 \times 6$ - and $3 \times 4$ - unit blocks in broken chain of non-zero blocks above the main diagonal of the matrix $S$.

\section{Example 3.}

Note that the permutation of the project jobs $\{a(i)\}_{i=1}^{5}$, as well as $\{a(i)\}_{i=6}^{14}$, does not change the accuracy of ordering. For example, a permutation of the jobs $\{a(i)\}_{i=6}^{14}$ of the Example 2 can get another $2 \times 2$-blocked $5 \times 9$-matrix of directly preceding of the jobs $\{a(i)\}_{i=6}^{14}$ by the jobs $\{a(i)\}_{i=1}^{5}$

$$
A[5 \times 9]=\left(\begin{array}{cccc:ccccc}
1 & 1 & 1 & 1 & 1 & 1 & 1 & 1 & 1 \\
1 & 1 & 1 & 1 & 1 & 1 & 1 & 1 & 1 \\
1 & 1 & 1 & 1 & 1 & 1 & 1 & 1 & 1 \\
\hdashline 0 & 0 & 0 & 0 & 1 & 1 & 1 & 1 & 1 \\
0 & 0 & 0 & 0 & 1 & 1 & 1 & 1 & 1
\end{array}\right),
$$

which, after the union of events (Fig. 4)

$v_{K}(a(1))=v_{K}(a(2))=v_{K}(a(3))=v_{H}(a(6))=v_{H}(a(7))=v_{H}(a(8))=v_{H}(a(9))$,

$v_{K}(a(4))=v_{K}(a(5))=v_{H}(a(10))=v_{H}(a(11))=v_{H}(a(12))=v_{H}(a(13))=v_{H}(a(14))$

and after the introduction of additional (fictional) job $b(1)$, stored directly preceding of the jobs $\{a(i)\}_{i=6}^{9}$ by the jobs $\{a(i)\}_{i=1}^{3}$, the jobs $\{a(i)\}_{i=10}^{14}$ by the jobs $\{a(i)\}_{i=4}^{5}$ and non-directly preceding and the jobs $\{a(i)\}_{i=10}^{14}$ by the jobs $\{a(i)\}_{i=1}^{3}$ corresponds to the following sub-boxes (Fig. 4).

Adding of the fictional job $b(1)$ between $a(5)$ and $a(6)$ does not modify other blocks of the matrix of direct precedence $\mathrm{S}$, establishing the correct sequencing united plurality of the jobs $b(1) \cup\{a(i)\}_{i=1}^{14}$ and turning the block $A[5 \times 9]$ to $2 \times 2$-blocked $6 \times 10$-submatrix 


\begin{tabular}{|c|ccccc:ccccc|}
\hline & $b(1)$ & $a(6)$ & $a(7)$ & $a(8)$ & $a(9)$ & $a(10)$ & $a(11)$ & $a(12)$ & $a(13)$ & $a(14)$ \\
\hline$a(1)$ & 1 & 1 & 1 & 1 & 1 & 0 & 0 & 0 & 0 & 0 \\
$a(2)$ & 1 & 1 & 1 & 1 & 1 & 0 & 0 & 0 & 0 & 0 \\
$a(3)$ & 1 & 1 & 1 & 1 & 1 & 0 & 0 & 0 & 0 & 0 \\
\hdashline$a(4)$ & 0 & 0 & 0 & 0 & 0 & 1 & 1 & 1 & 1 & 1 \\
$a(5)$ & 0 & 0 & 0 & 0 & 0 & 1 & 1 & 1 & 1 & 1 \\
$b(1)$ & 0 & 0 & 0 & 0 & 0 & 1 & 1 & 1 & 1 & 1 \\
\hline
\end{tabular}

with two $3 \times 5$-unit blocks in broken chain of the non-zero blocks above the main diagonal of the matrix $S$.

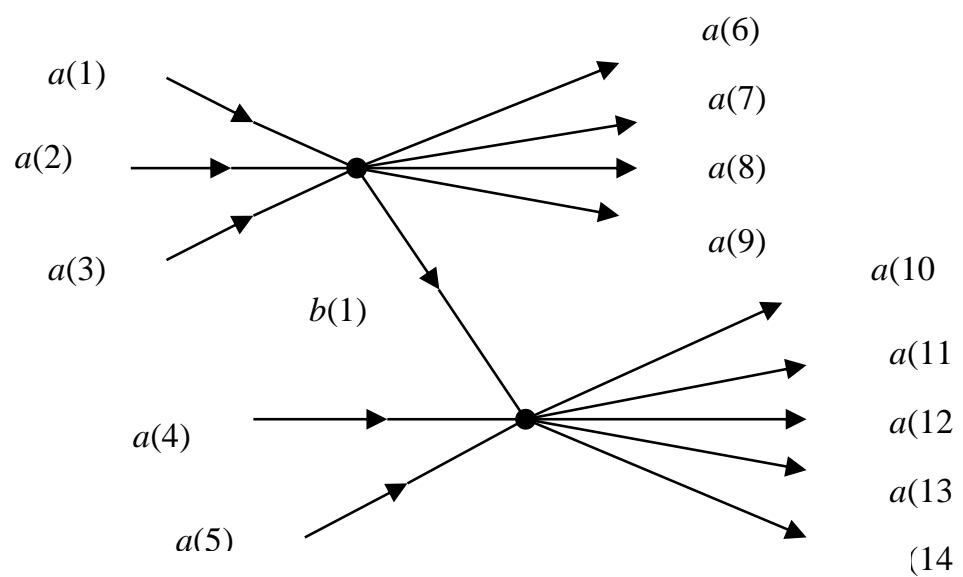

Fig. 4. - A graph constructed according to Example 3

\section{Algorithm of adding of the fictional jobs}

We give universal algorithm for adding the required number of fictional jobs $a(i) \notin P, i \geq k+1$.

Consider the non-zero non-trivial block $A[i \times j]$ of the rows $B \stackrel{d e f}{=}\{a(u)\}_{u=l}^{l+i}$ and the columns $C \stackrel{d e f}{=}\{a(u)\}_{u=l+i+1}^{l+i+j}, l=\sum_{u=1}^{t-1} i_{u}, l \leq i=i t, 1 \leq j=j t, 2 \leq t \leq r-1$, of the project jobs in the continuous chain $\left\{A\left[i_{t} \times j_{t}\right]\right\}_{t=1}^{r}$ blocked chained matrix of directly precedence $S$. We consider the set of previous project jobs $B$ as the factor-set of the relationship of equality of the rows of the matrix $A[i \times j]$. Then the relationship of direct sequence $s^{*}$ 1 determines the one-to-one mapping

$\mathrm{s}^{* \wedge}-1: 2^{\wedge} \mathrm{C} \rightarrow 2^{\wedge} \mathrm{C}$

on the set of all subsets of $C$. At the closure D of the image $s^{*^{-1}}\left(2^{C}\right)$ of her stepintersection subsets $\cap$ build the oriented tree of the relation

$\forall D_{1}, D_{2} \in D\left(D_{1 \rightarrow D 2} \stackrel{\text { def }}{\Leftrightarrow} D_{2} \subset D_{1}\right)$, 
excluding the empty set $\varnothing$. Arcs of this tree are the desired fictional jobs you needed to build the subgraph $G(A[i \times j])$, binding the project jobs $B \cup C$. Therefore, it completed the construction of sub-graph for non-trivial block in the Case 2.

As is a tree, the subgraph $G(A[i \times j])$ is planar, and the number of passing ways of the project $\mathrm{P}$ through the block $A[i \times j]$ is equal to $i \times j$.

Thus, the total number $M$ of the full paths of the project $P$ is $M=\prod_{t=1}^{r-1} i_{t}=\prod_{t=2}^{r} j_{t}$.

\section{Completion of construction of the project's graph}

Now from the resulting subgraphs $\left\{G\left(A\left[i_{t} \times j_{t}\right]\right)\right\}_{t=2}^{r-1}$ we may form the graph $G(V, R)$ of the project $P$. For this the initial vertex of starting jobs the initial vertex of the whole project, and the finishing jobs must be joined to the ending vertex of the project. Then all the end vertices of the block $A\left[i{ }^{1} \times j_{1}\right]$ will be the initial vertex of the block $A[i 2 \times j 2]$, and then to the endpoints of the block $A\left[i_{r-1} \times j_{r-1}\right]$, which became the initial vertex of the block $A\left[i r \times j_{r}\right]$.

This shows that, in any case, at least with the addition of some fictional jobs, the project's graph will be built.

\section{Reference}

1. Dokuchaev AV, Kotenko AP. Optimization of additional resources in network planning. Vestnik of Samara State Technical University. Series Sci. science, 2010; 1(20). [In Russian]

2. Dokuchaev AV, Kotenko AP. Transition to the direct precedence matrix for network project graph construction. Abstracts of VIII Conf. "Math. Simulation and Boundary Value Probl.", 2011; part 2. [in Russian]

3. Dokuchaev AV, Kotenko AP. Network project graph construction on the basis of jobs precedence table. Groups and Graphs, Algorithms and Automata-2015. Abstracts of the International Conf. Yekaterinburg: UrFU Publishing house, 2015.

4. Dokuchaev AV, Kotenko AP. Necessary conditions to complement the graph of project by fictitious arcs. Information Technology and Nanotechnology-2015. Abstracts of the International Conf. Samara: SSAU, 2015. [in Russian] 